\title{
A Practice-Based Analysis of an Online Strategy Game
}

\author{
Giannis Milolidakis ${ }^{1}$, Chris Kimble ${ }^{2}$, and Demosthenes Akoumianakis ${ }^{1}$ \\ ${ }^{1}$ Technological Education Institution of Crete, Greece \\ ${ }^{2}$ Euromed Management, Marseille, France \\ epp382@epp.teiher.gr, chris.kimble@euromed-management.com, \\ da@epp.teiher.gr
}

\begin{abstract}
In this paper, we will analyze a massively multiplayer online game in an attempt to identify the elements of practice that enable social interaction and cooperation within the game's virtual world. Communities of Practice and Activity Theory offer the theoretical lens for identifying and understanding what constitutes practice within the community and how such practice is manifest and transmitted during game play. Our analysis suggests that in contrast to prevalent perceptions of practice as being textually mediated, in virtual settings it is framed as much in social interactions as in processes, artifacts and the tools constituting the 'linguistic' domain of the game or the practice the gaming community is about.
\end{abstract}

Keywords: Online gaming, practice, social interaction, virtual worlds.

\section{Introduction}

In the early 1990s there was an upsurge of interest in on-line communities, stimulated in part by Howard Rheingold's book "The Virtual Community" [1] and in part by the growth of electronic networks such as the WELL (Whole Earth 'Lectronic Link). Multiplayer on-line games such as MUDS (Multiple User DungeonS) and MOOs (MUDS Object Oriented) were a focus of great deal of interest at the time. Now online multiplayer games are again attracting renewed attention. Although the earliest work in this area was concerned with issues of identity [2], later work began to focus on how on-line interaction takes place more generally and stressed the role played by social relationships [3]. More recent analyses of Massively Multiplayer Online Games [4] also makes use of social interaction and relationship building.

In this paper, we will continue this theme and analyze behavior in on-line games from a practice-oriented perspective informed by concepts from Communities of Practice [5], Networks of Practice [6] and Activity Theory [7]. In particular, we will examine the tools, processes and artifacts that allow the players to achieve their shared goals. Our analysis will centre on how the notion of practice is framed within the game and the ways in which this enables the players to interact, co-operate and compete. To this effect, we will employ what is sometimes termed virtual ethnography [8], to investigate the type, nature and scope of prevailing gaming practices and how they are framed and manifested during game play. 
The rest of the paper is structured as follows. The next section establishes the theoretical links of the present work by briefly addressing relevant concepts from the literature. Then, we present our research methodology and case study. Based on this the next section will discuss on-line gaming behavior from a practice oriented perspective. The paper concludes by summarizing key contributions.

\section{CoPs and NoPs}

The concept of a Community of Practice originated with Lave and Wenger [9] and was quickly adopted by other authors such as Brown and Duguid [10]. However, in much of this early work the notion of practice was left undefined beyond noting that practice is socially constructed and intimately connected to learning. Vann and Bowker [11] describe this early view as "an epistemology of practice that entails a set of claims about how people learn and how knowledge is shared among social actors". In his later work, Wenger developed the connection between practice and meaning arguing, "Practice is about meaning as an experience of everyday life" [5]. He explains that for the claims processors in his study, their 'practice' was something they had developed in order to make sense of their job. He also expanded the notion of what constitutes practice by introducing the idea that constellations of connected Communities of Practice could exist within a single organization. Later, Brown and Duguid [6] expanded this further by introducing the concept of Networks of Practice (NoPs) to describe groups of people who are geographically separate but who share similar interests or activities. NoPs share many of the features of CoPs but are organized at a more individual level and are based on personal rather than communal social networks. Using Granovetter's work on the strength of social ties, Brown and Duguid characterize NoPs as being linked by weak social ties.

The main difference between the two is that CoPs usually consist of people who know each other; they are primarily face-to-face communities that work closely together to accomplish their goals [12]. NoPs, on the other hand consist of people who may never get to know each other, but collaborate and transfer knowledge through the use of shared tools such as intranets [13]. Although they may develop similar identities, these are less tightly coupled to a NoP than would be the case with a CoP.

\section{Activity Theory and Virtual Worlds}

Activity Theory is a branch of Psychology that has a rich history of its own [7]; here we will simply highlight the points that are relevant to our own work. Briefly, Activity Theory argues that tools mediate human activity; when people interact to achieve some goal, they do so using tools. In Activity Theory, these tools are seen as an externalization of the internal knowledge of the toolmaker. As the knowledge of the toolmaker is 'embedded' in the tool, and as the tool mediates the activity, Activity Theory has become widely used in areas where a designer wishes to intentionally 'create' some activity, such as Human Computer Interaction [14].

Activity Theory is not a determinist theory: tools are remade and recast with use and new tools are created to deal with new situations. It does not argue that because 
knowledge is built into a tool in some way, and because a tool is sharable, then the knowledge used to create the tool also becomes sharable. The argument is more that tools condition certain patterns of actions and that by their repeated use these patterns become part of the accepted practice of the people who use the tools. This argument is more easily sustained in the world of CoPs and physical artifacts; when we move to the world of NoPs and 'virtual reality' however, the distinctions between the tool and patterns of action it conditions become harder to define. For example, in place-based social gatherings it is not possible for one person to deploy simultaneous multiple identities, whereas in virtual spaces tools to manage multiple identities are commonplace [15]. Thus, the practice of identity management in traditional settings is not simply reproduced in virtual space but is extended by the tools used to create that space. Similarly, civil inattention, the process by which we demonstrate awareness of one another in physical places [16], has no direct equivalent in virtual space, although awareness can be enhanced in other ways [17].

In light of the above, an obvious question to ask is what is actually happening in virtual spaces and how is practice encoded, enacted and transmitted online. The literature tends to focus on elements of practice framed in social interactions. Although this is valid, it fails to explain why certain offline practices are not reconstructed online or how it is that certain online practices do not have offline counterparts [13]. If practice is not simply reproduced online, but extended and enriched through digital media, then insights into this should improve our understanding of online behavior and offer a more appropriate unit of analysis for framing online practice.

\section{Case Study}

Travian is a massive multiplayer online strategic war game created by Travian Games $\mathrm{GmbH}$. Such games are relevant to the present work due to their inherent social connectivity, which classifies them both as interfaces to virtual spaces and communitysupport systems. Typically, communities are formed in the course of games to provide members with entertainment and/or online socialization using a dedicated repertoire of resources. Travian supports a 2D graphical environment and a messaging through which gamers can cooperatively attain individual and collective objectives, including private and public communication, diplomatic acts such as creation of alliances, declaration of war and the basic activities of warfare such as invasion, etc. The players' rewards, apart from entertainment, include socialization and the acquisition of a reputation within a community of online gamers [4].

The idea is that every new player becomes a major of a little village trying to attract (virtual) population by constructing and upgrading buildings and resource fields. New villages can be found or captured by players' joining alliances and coordinating their efforts. Winner of the game becomes the first alliance that will create a village containing a special building called "The World Wonder" and upgrade it to the highest level (i.e. 100). A village containing a "The World Wonder" needs enormous quantities of resources and a huge defense army to protect it against other alliances trying to destroy it. Achieving such a goal requires support of many players and consequently, it is nearly impossible for a single player to win. 


\subsection{Methods}

Two game characters were created in one of the many worlds (servers) of Travian as part of our virtual ethnography. One of the characters was less socially active than the other and did not have such an intense presence in the virtual world. Sufficient time was spent online to support the second character in an effort to join in a high standard and demanding alliance to team-up with experienced players and take part in non-trivial activities. Through daily participation in the virtual world and interaction with co-gamers, the active character established close relations to other players enabling liaisons with other game worlds through accessing characters from other servers. Our virtual ethnography was conducted with the first author being fully immersed in the game and observing how third-party game characters deal with their duties in the different settings (i.e. different worlds, different alliances). In a period of 11 months, a large file was compiled containing data about conversations in private and public chat rooms and forums or in the small discussion boards and chats. It is worth mentioning that at the end of the 11 month period, the "social" character owned 25 villages with more than 20 thousands of population in contrast to the 5 villages and the 4 thousands of population of the less social character.

\subsection{Research Questions}

Our analysis of the data, as presented below, aims to provide insight to two basic research questions: (a) what are the online practices underlying the socially intensive character's engagement (b) how are these practices manifested and intertwined during game play? Prior to the conducting the virtual ethnography, our working hypothesis was that practice is not necessarily framed only as social interaction, but may be embedded into artifacts, tools and processes through which collaborators create a history of co-engagement in the game's domain. Such co-engagement and its evolutionary nature may be used to explain why offline gaming practice is not merely reconstructed online, but frequently extended and expanded.

\section{Practice-Based Analysis of In-Game Behavior}

Activity theorists consider practice as subsuming activity [7]. Activity becomes meaningful for a designated practice through objects whose symbolic manifestation and relational properties are clearly defined and labeled. Moreover, activities are built on knowledge, skills or competences of those performing them. It is therefore important to relate practice to knowledge as expressed in communication acts or embodied into routines, procedures or patterns of use. Then, virtual practice in online gaming communities should be framed as much in communication acts - typically manifested as social interaction - as in the virtuality through which the virtual world is made sense of.

For Travian communities, social interaction is manifested primarily during game play as text messaging, posting or replying on a post. Members use tools to join/leave communities, express opinion, request support and negotiate options and strategies. For example, in order to join an alliance, messages must be sent from / to the leaders of the alliance asking for invitation. When the game world is in its early stages, the only criterion for finding good players to join in an alliance is the game statistics boards. The dialogue below gives an example of such interaction. 
DeathWing says: Good morning, would u like to join my alliance? We are impressed by your evolution.

DESTRO says: thanks for your invitation but I don't have embassy yet. I let you know \{Embassy: a building required to join an alliance

DeathWing says: ok then, I'm waiting msg from you when you are ready

This type of online socializing takes place outside the game board (i.e., the interactive manifestation of the game's virtual world) and frequently, without using the build-in communication mechanisms. In fact, high standard and demanding alliances make use of external tools, such as VOIP systems, external forums and blogs to communicate. Nevertheless, social interaction is strongly intertwined with game play. Specifically, making sense of online discussions will inevitably require knowledge of game's status and vice versa. For instance, consider the following narrative that presents a group message sent to all the members of an alliance.

Butterfly: Send 100 defend troops to the village (107 | -43) by midnight. Also send your hourly production to overcome the damages of the last attack \{hourly production: the production of all villages of a player in an hour\}

The message was sent using the build-in messaging system requiring from every player to send troops and resources to a specified village that was probably under attack. Players not familiar with the game may not immediately understand what is at stake. Consequently, narrative-based social interaction pre-supposes common ground on terms such as 'defend troops', 'hourly production', etc., which are tightly linked with the online game practice and its evolution. Eventually, such an intertwining between online game practices and social interaction leads to making sense of and coengaging in the game's virtual space. Nevertheless, each type of practice is shaped and served by different artifacts. Online game practice tends to explore visual, spatial and textural representations, while social interaction is textually mediated.

Travian uses visual artifacts to allow players to make sense of the virtual world and to convey social awareness. Specifically, a village, which occupies a square tile in the game map (Figure 1-a), provides the conceptual object for understanding the virtual world and inviting micro-negotiations between the players. As the notion of the village needs to be compatible with its physical counterpart, its virtual embodiment is depicted as a place-based territory inhabited by villagers. Specific functions of the village are manifested through visual artifacts. Resource fields (Figure 1-b) and buildings (Figure 1-c) define the economy and give extra capabilities to a village.

The player, through the process of upgrading the structural components of a village, increases the population of his/ her village and is able to use extra features of the game (i.e. training new types of troops). It is important to note that the choice of visual forms and their tractable information-processing properties determine the range of activities in which the user engages in and give meaning to otherwise banal actions such as mouse clicks, keystrokes and interaction sequences. For example, in order to create a new building a number of activities must be performed. The player selects an empty building spot (round tiles in the center of Figure 1-c) to raise it. A list of available buildings appears in the screen and then the player can select the desired 
building. Buildings take some time to complete but when they are ready, the player can upgrade them. By selecting a building of the village, a screen will appear providing information about the building, giving access to the capabilities of that building and allowing the upgrade.

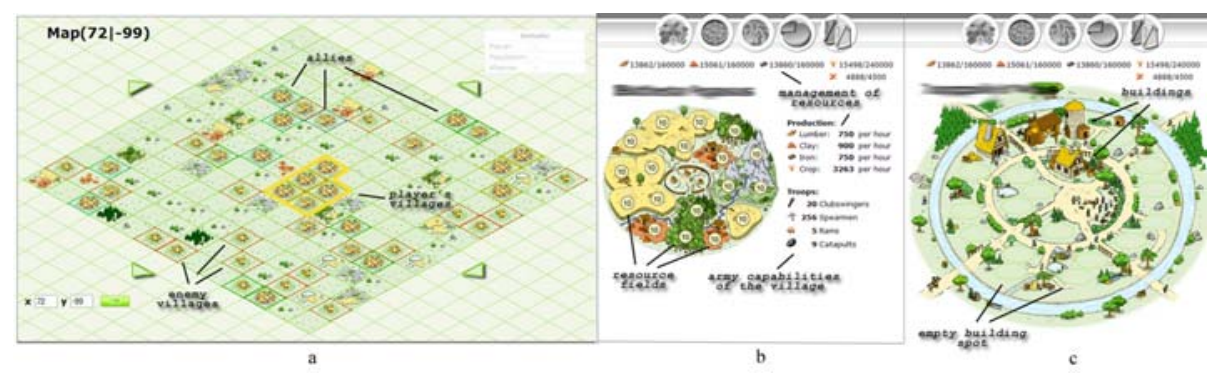

Fig. 1. a) Virtual world map b) Village overview, c) Village inner view

Another use of the visual artifacts offered by the game is to support social awareness. The game's map is used to represent who and what is around one player's village. In turn, this is used to determine tactics and drive social interactions. For example, depending on the setting (i.e., choice of tribes) the player explores the game map to identify possible enemies and / or allies. For a player with a big offensive army, other players with big offensive armies in the neighborhood are possible treats. This is because in order to keep and run such a big army a player needs to steal resources from other players nearby. Impediments to a player's development may occur when several other players seek to steal resources in the same area. On the other hand, such a player may take under his protection other players having defensive armies in order to get the extra protection from them. This, of course, influences the construction of alliances and who will be invited and included in them. Consequently, players with large armies find it difficult to coexist in the same alliance.

For a player it is important to know what exists near them; this becomes more important as the game world evolves in time and more collaborative duties need to be supported. The distinction of the allies in the game map is facilitated using colored tiles to represent the villages (see Figure 1-a). If a player wants to conquer a village then he / she must browse the game map to find out his / her nearby allies and ask for their help. An illustrative example is offered below.

Galactica says: Hi diamiano. Can u help me conquering the village of player $\mathrm{XXX}$ at (711-87)?

Damiano says: Yes why not? Do u want to conquer the village tonight?

Once commitment of a sufficient number of players is obtained, the group is invited to join in a discussion group in order to cooperate and achieve the target. A typical discussion concentrates on resource types, troop size, distance from target, etc., and takes place between participants who know enough each other and have a history of co-engagement in the alliance. 
Galactica says: Hi guys. As you know, I want to conquer the village at (711-87). How many available chiefs you have? \{Chiefs: type of troops needed to conquer a village Alinaki says: hi, I have 1 available and the necessary cp. \{cp: culture points, needed in order to conquer a village $\}$

Galactica says: ok I also have 3 \{chiefs $\}$... we need 2 \{chiefs $\}$ more

Damiano says: I have 2 chiefs available now and one more later tonight

Galactica says: ok 2 \{chiefs $\}$ will do the job

Galactica says: please give your exact travel times to the village (71I-87) \{a village is defined by its coordinates on the game map\}

In the extract below, the group discusses the tactical approaches for attacking and misleading their opponents. This entails coordinative assessment of a shared object of reference, namely the map, which offers social awareness by presenting the villages (friendly or not) taking part in this campaign.

Galactica says: I'm suggesting fake attacks to nearby villages f fake attack: an attack with one soldier

Galactica says: that is $(77,-65)(77,-62)(71,-64)(76,-66)$

Alinaki says: ok

Damiano says: one strike or multiple ones?

Galactica says: multiple waves \{multiple waves cause more damage to the enemy\}

Damiano says: ok

After negotiating and agreeing on the strategy, every player is aware of their respective duties in a specified time frame.

Galactica says: I will hit first on 23:59. Alinaki will hit on 00:00 and damiano some time real close after alinaki's hit. Is everyone ok with that?

Alinaki says: ok np

Damiano says: no problem for me too

Generalizing this workflow, we observe that players in their effort to accomplish their target formulate small groups, establish common ground by sharing information, negotiate options, devise plans for action and finally execute the plan. Through this process, gamers make sense of the virtual world, negotiate their tactics and reconstruct their individual and social standings.

\section{Conclusions}

Our analysis leads to several conclusions. Firstly, online gaming practices seem to subsume activities of peripheral domains of relevance such as online identity management, social networking and orchestration practices. Secondly, gaming practice is framed as much in social interaction as in the artifacts and tools embedded in and interactively manifested through the game. Both these are intertwined to determine making of sense, as well as the gamers' individual and collective behavior. Finally, online practice does not entail mere reproduction of offline gaming patterns. Several extensions were observed resulting from the digital medium and the gamers' history of co-engagement. The later point implies that the game's artifacts embody elements 
of practice to which users become accustomed because of their participation in the game. Thus, these artifacts and tools that are used to process them serve as the 'artificial' language for engagement in the linguistic domain of the game (i.e., gaming practice the community is about). The distinct characteristic of such language is that it should bridge the gap between the community and the virtual space, rather than the gap between humans and machines, as typically conceived by HCI researchers.

\section{References}

1. Rheingold, H.: The Virtual Community: Homesteading on the Electronic Frontier. Addison Wesley, Reading (1993)

2. Bruckman, A.S.: Gender Swapping on The Internet. In: Leiner, B. (ed.) International Networking Conference INET 1993, San Francisco (1993)

3. Conkar, T., Noyes, J.M., Kimble, C.: CLIMATE: A Framework for Developing Holistic Requirements Analysis in Virtual Environments. Interacting with Computers 11, 387-403 (1999)

4. Jakobsson, M., Taylor, T.L.: The Sopranos meets EverQuest: social networking in massively multiplayer online games. In: Proceedings of the 2003 Digital Arts and Culture (DAC), Melbourne, Australia, pp. 81-90 (2003)

5. Wenger, E.: Communities of Practice: Learning, Meaning, and Identity. Cambridge University Press, New York (1998)

6. Brown, J.S., Duguid, P.: The Social Life of Information. Harvard Business School Press, Boston (2000)

7. Engeström, Y., Miettinen, R., Punamäki, R.-L. (eds.): Perspectives on Activity Theory. Cambridge University Press, Cambridge (1999)

8. Hine, C.: Virtual Ethnography. SAGE Publications Ltd., Thousand Oaks (2000)

9. Lave, J., Wenger, E.: Situated Learning: Legitimate Peripheral Participation. Cambridge University Press, Cambridge (1991)

10. Brown, J.S., Duguid, P.: Organizational Learning and Communities of Practice: Toward a Unified View of Working, Learning, and Innovation. Organization Science 2, 40-57 (1991)

11. Vann, K., Bowker, G.C.: Instrumentalizing the truth of practice. Social Epistemology 15, 247-262 (2001)

12. Kimble, C., Hildreth, P.: Dualities, Distributed Communities of Practice and Knowledge Management. Journal of Knowledge Management 9, 102-113 (2005)

13. Vaast, E.: What Goes Online Comes Offline: Knowledge Management System Use in a Soft Bureaucracy. Organization Studies 28, 283-306 (2007)

14. Nardi, B.A.: Context and Consciousness: Activity Theory and Human-Computer Interaction. MIT Press, Cambridge (1996)

15. Jung, Y., Jin, S.A., McLaughlin, M.: Multiple Layers of Conjoint Action: Players' Identity Management in Role-Playing Blogs. In: Annual meeting of the NCA 93rd Annual Convention, Chicago (2007)

16. Goffman, E.: Behaviour in Public Places. Notes on the Social Organization of Gatherings. Free Press, New York (1966)

17. Dourish, P., Bly, S.: Portholes: supporting awareness in a distributed work group. In: CHI 1992: Proceedings of the SIGCHI conference on Human factors in computing systems, p. 547. ACM Press, New York (1992) 